\title{
Quality of Transmission Impact on Routing and Wavelength Assignement Rules in Hybrid Optical Networks
}

\author{
Imen OUARDA and Mourad MENIF \\ Electronic, Physics and Propagation Department \\ École Supérieure des Communications de Tunis \\ Cité Technologique des Communications, Rte de Raoued \\ Km 3.52083 - Ariana - Tunisia \\ \{imen.ouarda, mourad.mnif\}@supcom.rnu.tn
}

\author{
Mohamed KOUBAA and Maroua BAKRI \\ Communications and Information Technologies Department \\ École Nationale d'Ingénieurs de Tunis \\ BP 37 - Le Belvédère 1002 - Tunis - Tunisia \\ \{mohamed.koubaa, Maroua.bakri\}@enit.rnu.tn
}

\begin{abstract}
Transmission quality is being a very important issue in optical communication systems. As an optical signal propagates along a lightpath connecting a source node to a destination node in the network, the quality of transmission is degraded due to transmission impairments introduced by long-haul optical components. Consequently, the signal's Bit Error Rate (BER) at the destination node can become unacceptably high. It is, thus, necessary to find an efficient method to estimate these impairments penalties and to evaluate the optical links transmission quality. In this paper, an analytic expression of the Q-factor is presented. To the best of our knowledge, this is the first time that the Q-factor estimation is presented in details considering four main impairments referred to as Chromatic Dispersion (CD), Polarization Mode Dispersion (PMD), Optical Signal to Noise Ratio (OSNR) and Nonlinear Phase $\left(\Phi_{N L}\right)$.
\end{abstract}

Transmission quality, Q-factor, Chromatic Dispersion, Polarization Mode Dispersion, Optical Signal to Noise Ratio, Nonlinear Phase.

\section{INTRODUCTION}

In WDM networks, to set up a traffic request, two processes must be achieved namely the routing process and the wavelength assignment process. In the former, a physical path, connecting the source node to the destination node of the considered traffic request, is calculated. While, in the latter, one or several wavelengths are assigned to each link of the computed path. The problem of traffic demand establishment is, generally, known as the Routing and Wavelength Assignment (RWA) problem. Many algorithms resolving the RWA problem have been developed in the literature [1-4]. Most of them assume an ideal optical medium. Physical layer effects are hence totally neglected and significant transmission physical phenomena have been ignored. In real case, many linear and nonlinear physical layer impairments can seriously disturb the optical signal quality. This may lead to the rejection of the traffic request whenever the optical communication quality goes under a specified limit.

This paper addresses the transmission quality problem in WDM networks rather than the usual RWA problem. We focus on how to estimate the optical communication quality along a physical path by computing its Q-factor. This is done in an analytic way considering four main physical layer impairments which are respectively: Chromatic Dispersion (CD), Polarization Mode Dispersion (PMD), Optical Signal to Noise Ratio (OSNR) and Nonlinear Phase $\left(\Phi_{N L}\right)$.

The rest of the paper is organized as follow. In Section II, some Q-factor expressions, found in the literature, are presented. Section III is dedicated to describe the problem dealt with in this paper. In Section IV, the adopted analytical Qfactor expression is detailed. Simulations results are discussed in Section V. The main contributions of the paper are, finally, summarized in Section VI.

\section{RELATED WORKS}

The performance of an optical communication system is measured by the Bit Error Ratio (BER), which is the ratio between the number of defected bits arriving at the receiver and the total number of transmitted bits [5]. In very high bit rate systems, such as WDM networks, computing BER requires many simulation executions and a long computation time. Instead of BER, the system performance can be expressed in terms of Q-factor, which is related to BER through the following equation [5-7]:

$$
B E R=\frac{1}{2} \operatorname{erfc}\left(\frac{Q}{\sqrt{2}}\right)
$$

where $\operatorname{erfc}$ is the complementary error function.

The Q-factor estimation constitutes today a challenging topic which attracts many recent research works dealing with the transmission quality problem in WDM networks.

Currently, several Q-factor expressions have been presented in the literature, however, the common definition was [5][7]: 


$$
Q=\frac{\mu_{1}-\mu_{0}}{\sigma_{1}+\sigma_{0}}
$$

where $\mu_{1}$ and $\mu_{0}$ are respectively the bit "one" and "zero" average powers. $\sigma_{1}$ and $\sigma_{0}$ are respectively their standard deviations.

In [5] and [8], a relationship between Q-factor and OSNR is defined as according to Equation 3:

$$
Q=\frac{O S N R}{\sqrt{O S N R+1}+1} \sqrt{\frac{B_{0}}{B_{e}}}
$$

where $B_{e}$ is the electrical bandwidth and $B_{0}$ is the optical bandwidth. Ignoring some physical impairments, such as CD, PMD and $\Phi_{N L}$, when computing the Q-factor, may lead to unreliable performance estimation.

\section{DESCRIPTION OF THE PROBLEM}

Once the RWA problem is resolved, the optical communication quality through each computed lightpath should be measured. A lightpath is then rejected if its quality is poor or under some specific limit. In such case, a second lightpath must be computed to carry data traffic. The lightpath quality is evaluated by analytically estimating its corresponding Q-factor considering the power penalties of each of the four aforementioned impairments (CD, PMD, OSNR, $\Phi_{N L} \Phi$.

In this paper, a lightpath corresponds to a set of optical segments. An optical segment connects two consecutive OXCs and is composed of at least one span. Each span is a $70 \mathrm{~km}$ optical section followed by an erbium-doped fiber amplifier (EDFA) and a chromatic dispersion compensation module. The $70 \mathrm{~km}$ optical section is made up of transmission fiber, Single Mode Fiber (SMF) or Non Zero Dispersion Shifted Fiber (NZDSF).. The Dispersion Compensation Fiber (DCF) represents the chromatic dispersion compensation module. A typical configuration of an optical segment is depicted in Figure 1.

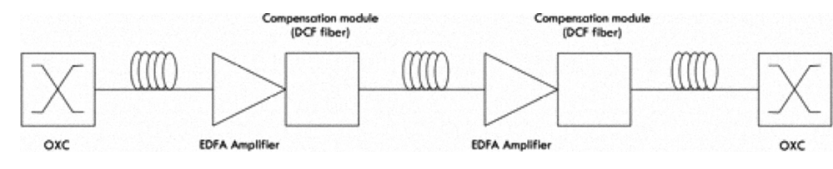

Figure 1. A typical configuration of an optical segment

\section{Q-FACTOR COMPUTATION}

\section{A. Chromatic Dispersion (CD)}

Chromatic dispersion is mainly caused by the optical source non null spectral width. Several spectral components constitute the pulse to be injected in the fiber; each one is traveling through the lightpath with its speed or its group velocity. Difference in velocities induces a delay known as the Group Velocity Delay (GVD). The GVD itself causes the pulse to be broadened over the time domain [9] leading to Inter-Symbol Interference (ISI) and energy decrease.

The direct consequence of energy decrease is the signal to noise ratio (SNR) degradation at the decision circuit. In order to maintain the ratio at an acceptable level, the required average power must be important at the receiver. This, in fact, is the $\mathrm{CD}$ penalty origin. $\mathrm{As} C D$ is considered as a deterministic effect, its penalty is estimated by the Eye Opening Penalty (EOP) expressed in $\mathrm{dB}$. CD penalty, noted $E O P_{C D}$, is given by the following equation [10]:

$$
E O P_{C D}=10 \log \left(f_{b}\right)
$$

where $f_{b}$ is the broadening factor; the ratio between the pulse temporal width, $\sigma$, after travelling along the lightpath, and the initial temporal width $\sigma_{0}$. The broadening factor is defined as follow [10]:

$$
f_{b}=\frac{\sigma}{\sigma_{0}}=\sqrt{1+\left(C D_{\text {total }} \frac{\sigma_{\lambda}}{\sigma_{0}}\right)^{2}}
$$

where $C D_{\text {total }}$ is the total cumulated chromatic dispersion at the end of the lightpath expressed in $\mathrm{ps} / \mathrm{nm}$ and $\sigma_{\lambda}$ is the pulse temporal width, related to the Full Width at Half Maximum (FWHM) [11].

\section{B. Polarization Mode Dispersion (PMD)}

The optical signal travelling through the fiber has two orthogonal Principal States of Polarization (PSP) that in ideal case will have the same speed. However, fiber symmetry default causes a signal random birefringence [5]. This leads to a delay between the PSP, called Differential Group Delay $(D G D)$. PMD causes pulse broadening leading to ISI increase. PMD is also considered as a deterministic effect. Its penalty is, again, estimated by the EOP and is given by [9]:

$$
E O P_{P M D}=A\left(\frac{\Delta \tau}{T_{B}}\right)^{2} \gamma(1-\gamma)
$$

where $A$ is a pulse form factor depending on the pulse form and the receiver characteristics. $\gamma$ is the power splitting; its value is in general between 0 and $1 . T_{B}$ is the time bit and $\Delta \tau$ is the PMD quantity expressed in $p s$.

To evaluate the lightpath's PMD penalty, it is necessary to compute the cumulated PMD at its end, $\Delta \tau_{\text {total }}$ given by [6]:

$$
\Delta \tau_{\text {total }}=\sqrt{\sum_{\text {segment } \epsilon \text { path }}\left(\Delta \tau_{\text {segment }}\right)^{2}}
$$


where $\Delta \tau_{\text {segment }}$ is the cumulated PMD at the end of a lightpath's segment expressed as follow :

$$
\begin{gathered}
\Delta \tau_{\text {segment }}=\sqrt{\sum_{\text {span } \epsilon \text { segment }}\left(\Delta \tau_{\text {span }}\right)^{2}} \\
=\sqrt{\sum_{\text {span } \epsilon \text { segment }}\left(P M D_{\text {coef }} \sqrt{L_{\text {span }}}\right)^{2}}
\end{gathered}
$$

where $\Delta \tau_{\text {span }}$ is the cumulated PMD at the end of a segment's span, $L_{\text {span }}$ is the span length and $P M D_{\text {coef }}$ is the fiber PMD coefficient. PMD is in fact caused by the transmission fiber (SMF and NZDSF) and the dispersion compensating fiber (DCF).

\section{Optical Signal to Noise Ratio (OSNR)}

In order to preserve optical signals from different types of degradation and to ameliorate their reach, Erbium-Doped Fiber Amplifiers (EDFA) are used in optical networks. The amplification process comes with, inevitably, Amplified Spontaneous Emission (ASE). The ASE is generated at every amplification stage and is amplified through the lightpath. In the worst case, the noise is so important that it omits the useful signal.

Optical Signal to Noise Ratio is the measure of the ratio of signal power to noise power. The noise power corresponds essentially to ASE power. The amplifier's OSNR is given by [12]:

$$
O S N R=\frac{P_{\text {out }}}{P_{A S E}}=\frac{P_{\text {out }}}{2 n_{s p}(G-1) h \frac{C}{\lambda} \Delta f}
$$

where $P_{\text {out }}$ is the amplifier output power, $C$ is the light celerity, $h$ is Planck's constant, $\Delta f$ is the optical bandwidth, $G$ is the optical gain, $\lambda$ is the signal wavelength and $n_{s p}$ stands for the inversion population factor expressed as [12]:

$$
n_{s p}=0.5 \times 10^{\frac{N F}{10}}
$$

where $N F$ is the amplifier noise figure.

The OSNR obtained at the end of a lightpath is calculated by considering OSNRs computed for each segment composing the lightpath according to the following expression [6][12]:

$$
\frac{1}{O S N R_{\text {final }}}=\sum_{i} \frac{1}{\text { OSNR }_{\text {segment }_{i}}}
$$

where $O S N R_{\text {segment }}$ is the OSNR of a lightpath's segment. The segment's OSNR is computed by considering the OSNRs of the spans composing the considered segment:

$$
\frac{1}{O S N R_{\text {segment }}}=\sum_{i} \frac{1}{O S N R_{\text {span }_{i}}}
$$

\section{Nonlinear Phase $\left(\Phi_{N L}\right)$}

The nonlinear phase is the direct consequence of the Kerr effect. An electromagnetic field causes the fiber refractive index change and consequently a phase shift. This leads to a bit rate proportional ISI. The nonlinear phase is expressed as follow [12]:

$$
\Phi_{N L}=\gamma L_{e f f} P_{i n}
$$

Where $P_{\text {in }}$ the input power, $\gamma$ is is the nonlinearity coefficient, and $L_{e f f}$ is the fiber effective length expressed as follows:

$$
L_{e f f}=\frac{1-e^{-\alpha L}}{\alpha}
$$

The nonlinearity coefficient is given by:

$$
\gamma=\frac{n_{2} \omega_{0}}{A_{e f f} C}=\frac{2 \pi n_{2}}{\lambda_{0} A_{e f f}}
$$

where $n_{2}$ is the fiber nonlinearity index, $\lambda_{0}$ is the wavelength traveling through the fiber and $A_{\text {eff }}$ is the fiber effective area.

In compliance with other physical phenomena, the nonlinear phase shift causes a power penalty. A typical value of $1.5 \mathrm{~dB}$ is used to estimate the nonlinear phase shift penalty. This value is equivalent to 1 radian phase shift [13].

\section{E. Q-factor estimation}

For a preliminary estimation of the lightpath quality, we assume that the dominant noise in WDM networks is the ASE channel beat noise. For this reason, we consider that the noise variance on the bit "one" is only given by the signalspontaneous noise variance. While, the noise variance on the bit "zero" is supposed to be negligible.

$$
\sigma_{1}^{2}=\sigma_{\text {sig-sp }}^{2}=4 \times P_{\text {avg }} \times P_{A S E} \times \frac{\Delta f_{\text {elec }}}{\Delta f_{\text {opt }}}
$$

where $P_{a v g}$ is the average optical power and $P_{A S E}$ is the ASE power, $\Delta f_{\text {opt }}$ is the optical bandwidth and $\Delta f_{\text {elec }}$ is the electrical bandwidth.

The eye opening of the optical signal, expressed by the difference $\mu_{1}-\mu_{0}$, can be approximated by the average optical power: 


$$
\mu_{1}-\mu_{0} \approx 2 \times P_{\text {avg }}
$$

Considering Equation 2 and taking into account Equation 16 and Equation 17, the Q-factor estimation considering only the ASE noise is then expressed as follows:

$$
Q=\sqrt{\frac{O S N R \times \Delta f_{\text {opt }}}{\Delta f_{\text {elec }}}}
$$

where the OSNR expression is given by:

$$
O S N R=\frac{P_{\text {avg }}}{P_{A S E}}
$$

The linear and non linear phenomena also affect the lightpath quality. Their penalties reduce the inner eye opening, and lead to changes in the noise variance which decrease hence the Q-factor.

The linear effects, such as $\mathrm{CD}$ and $\Phi_{N L}$, are taken into account by considering their respective EOPs. The total penalty is then integrated in the Q-factor estimation. The additional penalty due to first-order PMD, the nonlinear effect, is thereafter separately added. Here, it is assumed that the PMD only degrades the inner eye opening and does not cause changes in the noise variance.

We thus define the analytic way to define the lightpath Qfactor based on OSNR, PMD, CD and $\Phi_{N L}$ according to the following expression [9]:

$$
Q=\frac{1}{E O P_{P M D}}\left(\sqrt{\frac{O S N R}{E O P_{C D} E O P_{\Phi_{N L}}} \frac{\Delta f_{\text {opt }}}{\Delta f_{\text {elec }}} E X T P}\right)
$$

where $O S N R$ is the lightpath final OSNR, EOP $P M D$ is the PMD penalty, $E O P_{C D}$ is the $\mathrm{CD}$ penalty and $E O P_{\Phi_{N L}}$ is the nonlinear phase penalty computed at the end of the lightpath (destination node). EXTP stands for the extinction ratio penalty. It corresponds to the ratio between the power $P_{1}$ (bit "one") and the power $P_{0}$ (bit "zero") [14].

$$
r_{e}=\frac{P_{1}}{P_{0}}
$$

Theoretically, $P_{0}$ should be zero to get an infinite $r_{e}$. However in most optical transmitters, $P_{0}>0$ and the $r_{e}$ value is not optimal. This, in fact, is the extinction ratio penalty origin which is defined as follow [14]:

$$
E X T P=\frac{P_{a v g}\left(r_{e}\right)}{P_{a v g}\left(r_{e}=\infty\right)}=\frac{r_{e}+1}{r_{e}-1}
$$

\section{SIMULATION RESUlTS}

In this section, we carried out several simulation experiments and analyzed the observed results. Two transmission fiber types are considered namely SMF and NZSDF. The optical signal amplification is provided by EDFA amplifier and DCF fibers are used for chromatic dispersion compensation. We consider 40 channels (wavelengths) at $100 \mathrm{GHz}$ spacing around $1550 \mathrm{~nm}$. Parameters values used for the experiments are listed in Table 1. In the following a value of $6(7.8 \mathrm{~dB})$ is fixed as the lowest limit for the Q-factor $\left(Q_{\text {threshold }}\right)$. This limit corresponds to $10^{-9}$ for the BER [10].

\section{A. Validation}

We first studied the behavior of the Q-factor along a pointto-point optical link. We varied the length of the considered link and observed the penalty of each transmission impairment and then estimated the Q-factor. SMF fibers at $10 \mathrm{Gbps}$ are used.

TABLE I. TRANSMISSION SYSTEM PARAMETERS [6]

\begin{tabular}{|l|c|}
\hline PARAMETER & VALUE \\
\hline SMF chromatic dispersion $(\mathrm{ps} / \mathrm{nm} . \mathrm{km})$ & 17.0 \\
\hline SMF polarization mode dispersion $\left(\mathrm{ps} / \mathrm{km}^{1 / 2}\right)$ & 0.1 \\
\hline SMF losses $(\mathrm{dB} / \mathrm{km})$ & 0.2 \\
\hline NZDSF chromatic dispersion $(\mathrm{ps} / \mathrm{nm} . \mathrm{km})$ & 4.3 \\
\hline NZDSF polarization mode dispersion $\left(\mathrm{ps}^{\prime} / \mathrm{km}^{1 / 2}\right)$ & 0.1 \\
\hline NZDSF losses $(\mathrm{dB} / \mathrm{km})$ & 0.22 \\
\hline DCF chromatic dispersion $(\mathrm{ps} / \mathrm{nm} . \mathrm{km})$ & -90.0 \\
\hline DCF losses $(\mathrm{dB} / \mathrm{km})$ & 0.6 \\
\hline DCF polarization mode dispersion $\left(\mathrm{ps} / \mathrm{km}^{1 / 2}\right)$ & 0.4 \\
\hline
\end{tabular}

Figure 2 shows the variation of the Q-factor with respect to distance. Figure 2(a) (respectively Figure 2(b)) plots the Qfactor variation when no CD compensation modules are used (respectively when considering $\mathrm{CD}$ modules). The figure depicts impairments accumulation effect on the Q-factor. The Q-factor decreases when the distance between end-nodes increases and reaches critical values for important distances (above $800 \mathrm{Km}$ ). Without considering the CD compensating modules, an important difference is observed between the curve of Q-factor when considering only OSNR and when both OSNR and CD are considered. For the other curves, close values have been obtained. It is obvious that $C D$ is the most penalizing impairment for the transmission quality.

Distinct improvement is observed when chromatic dispersion compensating modules are added. We noticed that the values of 
the Q-factor computed by only considering OSNR are reduced. This can be explained by the fact that the delivered EDFA gain is slightly increased passing from 14 to $22 \mathrm{~dB}$. This increase is required to compensate the loss of the DCF modules. In spite of this reduction, the Q-factor values computed when considering all transmission impairments are still high.
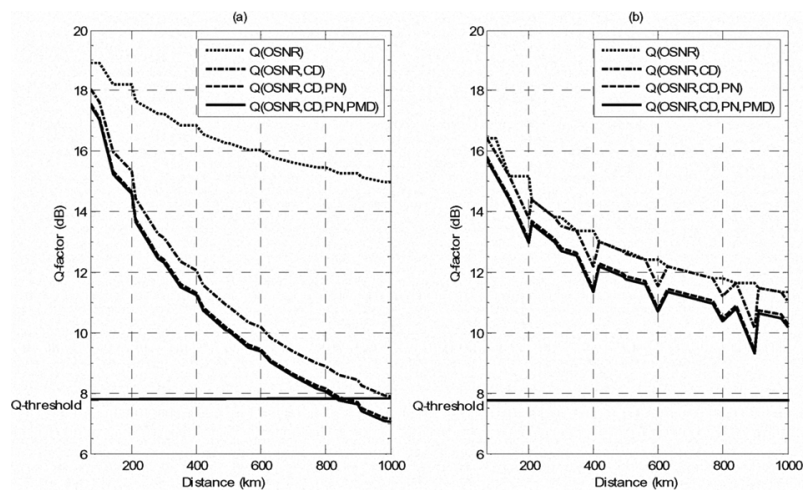

Figure 2. Variation of the Q-factor with respect to distance: (a) without CD compensation modules. (b) with CD compensation modules

\section{B. Study cases}

In this section, we discuss the impact of optical signal transmission quality on the lightpath blocking due to nonadmissible Q-factor. Let us remind that the Q-factor is computed by considering transmission impairments accumulations along a lightpath from its source node to its destination node. For this purpose, we consider two different network topologies: the European Backbone Network (EBN) and the National Science Foundation Network (NSF) shown in Figure 3 and Figure 4 respectively. The EBN network is composed of 57 nodes interconnected with 170 links. The shortest link is $26 \mathrm{~km}$ long while the longest is $680 \mathrm{Km}$. The NSF network is composed of 18 nodes interconnected by 58 links where the shortest is $400 \mathrm{~km}$ long and the longest is 2400 $\mathrm{km}$.

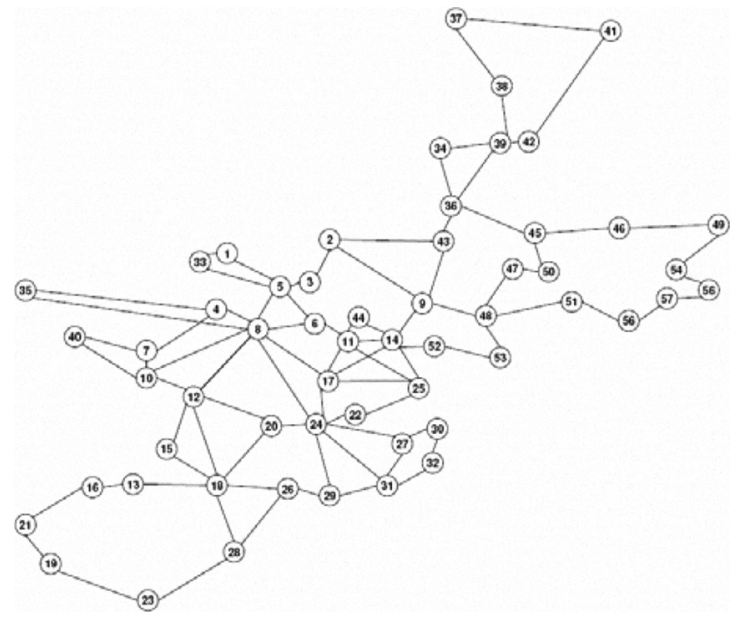

Figure 3. The EBN network topology

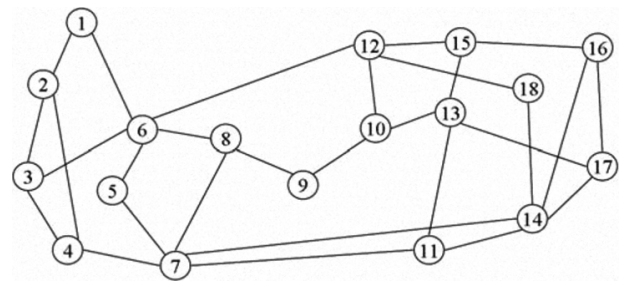

Figure 4. The NSF network topology

To investigate the effect of linear impairments on the quality of transmitted signal, we computed the shortest path (in terms of distance) for each possible source-destination pair in the network according to the algorithm described in [16]. Each path is a potential candidate route to carry a traffic request. A total number of 3192 paths are computed on the EBN network where the longest path is $4662 \mathrm{~km}$ long. Only 306 paths are computed for the NSF network where the longest path is $5700 \mathrm{~km}$. We then computed the Q-factor for each shortest path along the central wavelength. A lightpath with a Q-factor lower than the fixed threshold $\left(Q_{\text {threshold }}\right)$ is considered as a non-admissible lightpath and hence cannot be used to carry a traffic request. We used once again SMF fibers at $10 \mathrm{Gbps}$ and assumed that EDFA are spaced by $70 \mathrm{~km}$. The EDFA gain is fixed at $14 \mathrm{~dB}$ required to recover the losses induced by fiber-spans. The obtained Q-factors values are drawn in Figure 5 assuming no CD compensating modules in the network.

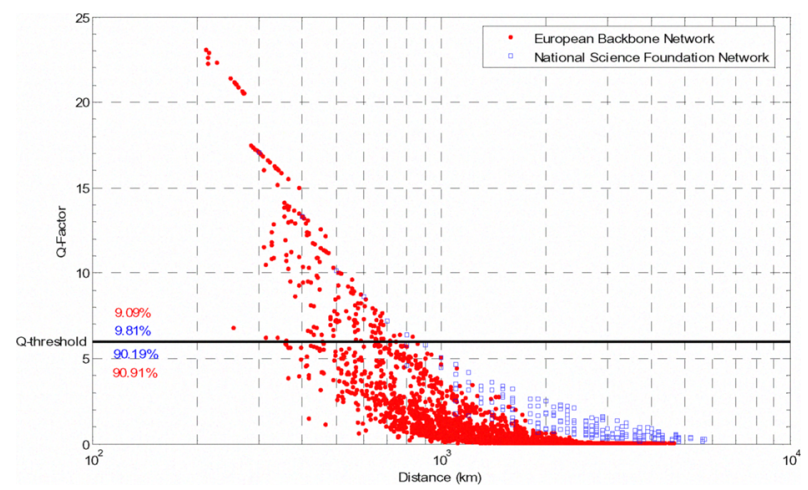

Figure 5. Q-factor with respect to distance (link at $10 \mathrm{Gbps}$ using SMF without CD compensation)

One notices that most of lightpaths present a Q-factor that exceeds the fixed lowest limit. Indeed $90.91 \%$ of the lightpaths on the EBN network and $90.19 \%$ of the lightpaths on the NSF network have Q-factors lower than the admissible one.

In Figure 6, we reported the computed Q-factors in the presence of dispersion compensating modules. We used 13.2 $\mathrm{km}$ long DCF fibers inducing an increase of the EDFA gain to $22 \mathrm{~dB}$. The ratio of lightpaths presenting a Q-factor lower than the admissible threshold is still important. In fact, $88.97 \%$ of the lightpaths on the EBN network and $68.95 \%$ of the 
lightpaths on the NSF network have non-admissible Q-factors. A minor improvement has been observed for the EBN network. This is due to the fact that for the same travelling distance, the optical signal passes through more links in the EBN network than in the NSF network.

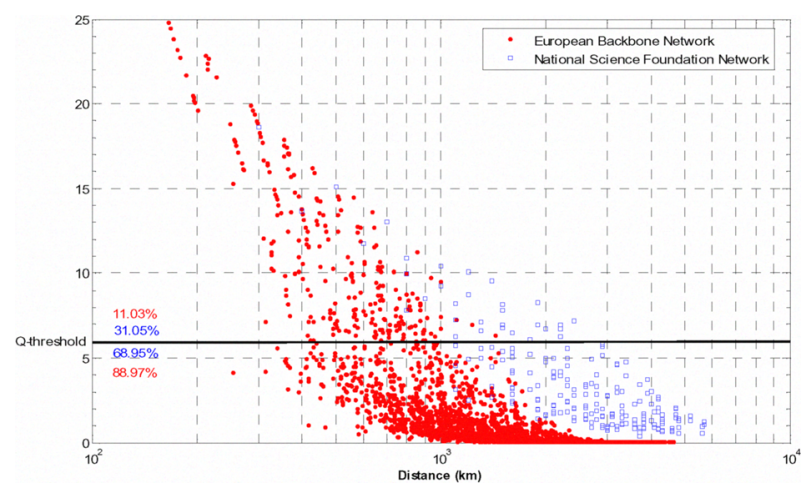

Figure 6. Q-factor with respect to the distance (link at $10 \mathrm{Gbps}$ using SMF with CD compensation modules).

Still considering the same simulation experiment, we now aim at investigating the impact of the fiber-type on lightpath blocking. NZDSF fibers are used. This leads to a reduction in the necessary length of DCF fibers to only $1.88 \mathrm{~km}$ and the required EDFA gain is only $17 \mathrm{~dB}$. Figure 7 shows the new distribution of the Q-factor values.

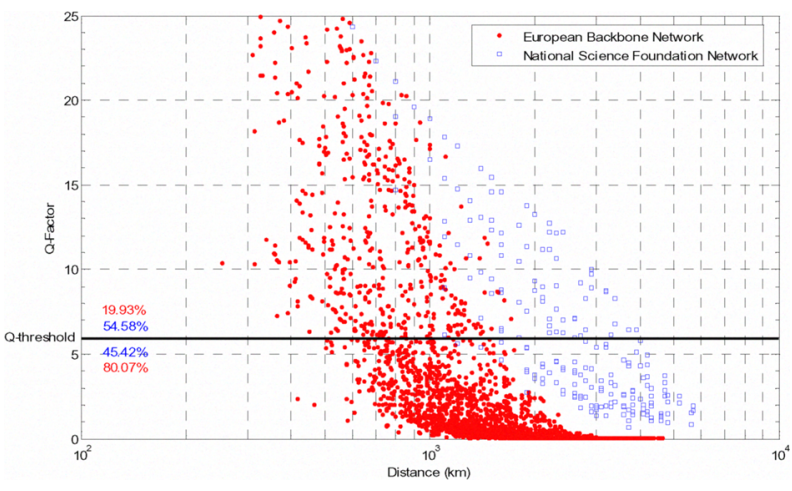

Figure 7. Q-factor with respect to distance (link at $10 \mathrm{Gbps}$ using NZDSF with CD compensation modules)

One observes that when using NZDSF fibers the ratio of non-admissible lightpaths goes from $90.91 \%$ to $80.07 \%$ for the EBN network. For the NSF network, the same ratio goes from $90.19 \%$ to $45.42 \%$.

In spite of using NZDSF fibers with DCF compensating modules, the ratio of non-admissible lightpaths is still high especially on the EBN network. In fact, this result emphasizes on our previous remark concerning the links length on the NSF network.

To overcome the severe lightpath blocking due to signal quality degradation, we here studied the impact of signal regeneration on the ratio of non admissible lightpaths. For this purpose, we placed regenerators at each node in the network and observed once again the ratio of non-admissible lightpaths on the EBN and the NSF networks at 10 and 40 Gbps (see Table II).

TABLE II. RATIO OF NON-ADMISSIBLE LIGHPATHS FOR DIFFERENT SCENARIOS AT 10 AND 40 GbpS FOR EBN AND NSF NETWORKS

\begin{tabular}{|l|c|c|c|c|}
\hline \multirow{2}{*}{ SCENARIO } & \multicolumn{2}{|c|}{ EBN } & \multicolumn{2}{c|}{ NSF } \\
\cline { 2 - 5 } & $10 \mathrm{Gbps}$ & $40 \mathrm{Gbps}$ & $10 \mathrm{Gbps}$ & $40 \mathrm{Gbps}$ \\
\hline 1: SMF w/o DCM, w/o 3R & $90.91 \%$ & $95.11 \%$ & $90.19 \%$ & $98.69 \%$ \\
\hline 2: SMF w DCM, w/o 3R & $88.97 \%$ & $93.85 \%$ & $68.95 \%$ & $85.29 \%$ \\
\hline 3: NZDSF w DCM, w/o 3R & $80.07 \%$ & $85.11 \%$ & $45.42 \%$ & $55.22 \%$ \\
\hline 4: NZDSF w DCM, w 3R & $0 \%$ & $0 \%$ & $13.07 \%$ & $65.06 \%$ \\
\hline
\end{tabular}

The regeneration contribution is immediate. Only $13 \%$ of the lightpaths on the NSF network still have Q-factor lower than the fixed threshold whereas all the lightpaths on the EBN network present a Q-factor at least equal to the fixed threshold. One notices that the number of non-admissible lightpaths is still positive for the NSF network even though all the network nodes have regeneration capabilities. This is due to the fact that some optical links are too long that even with regeneration at each node, lightpath Q-factor goes under the $Q_{\text {threshold }}$. Thus, placing regenerators on the link between its end-points becomes necessary. Table II also shows that the number of non-admissible lightpaths recoded at $40 \mathrm{Gbps}$ is more critical than the ones recoded at $10 \mathrm{Gbps}$. This emphasizes the impact of the bit rate on the transmission quality. In fact, as the bit rate is the reciprocal of the time bit, low time bit leads to more critical penalties for CD and PMD.

Thanks to optical regeneration, it has been shown that the ratio of blocked lightpaths is reduced to its minimum. Nevertheless, placing regenerators at each intermediate node in the network should be too costly for the network deployment and remains inefficient for some topologies as outlined in the preceding section for the NSF network. In order to manage the cost deployment of future WDM networks, it becomes imperative to optimize the placement of regenerators.

\section{CONCLUSIONS}

This paper has focused on transmission quality in WDM networks. A precise estimation of the optical lightpath transmission quality by including the maximum of impairments makes the problem of routing and wavelength assignment (RWA) more reliable regarding to the rejection of a traffic request due to non-admissible Q-factor. For this purpose, an analytic expression computing the Q-factor along a lightpath in a WDM network is given. Four main linear physical impairments have been considered in the Q-factor estimation; chromatic dispersion (CD), polarization mode dispersion (PMD), optical signal to noise ratio (OSNR) and nonlinear phase $\left(\Phi_{N L}\right)$. Two different network topologies are considered for the experiments: the European Backbone 
Network (EBN) and the National Science Foundation Network (NSF). For each network, the first shortest path between each source-destination pair is computed and its Q-factor is estimated for the central wavelength $(1550 \mathrm{~nm})$. By considering various scenarios, the ratio of non-admissible lightpaths (lightpaths with Q-factor lower than a fixed threshold) is analyzed. Several parameters have been considered for the experiments such as the type of the transmission fiber (SMF and NZDSF), the bit rate (10 and 40 Gbps) and the possibility to use regeneration. Through the obtained results, we have proved that among the considered linear impairments, chromatic dispersion is the most penalizing for the transmission quality. The performances, in terms of number of non-admissible lightpaths, are improved by using NZDSF fibers. Moreover, the use of regenerators enhances the reach of optical signals and hence reduces the ratio of non-admissible lightpaths. It has been shown that even by placing a regenerator at each node on the NSF topology, some lightpaths still have poor Q-factors. It is essential then to provide both minimal-cost and heuristic algorithms for the optimal placement of regenerators.

In this article, we have been interested on the quality of transmission for a static traffic request by considering only the linear impairments. For our future studies, we will consider the case of dynamic traffic by including in the Q-factor estimation the penalties of nonlinear impairments such as the nonlinear effects of the transmission fiber (FWM, XPM ...) and the crosstalk generated when going through optical components (EDFA, OXC ...). We will also investigate the appropriate approach for the placement of regenerators in WDM networks.

\section{REFERENCES}

[1] H. Zang, J.P. Jue, and B. Mukherjee, "A review of routing and wavelength assignment approaches for wavelength-routed optical WDM networks," SPIE Optical Networks Magazine, Vol. 1, no. 1, pp.
47-60, Jan. 2000.

[2] A.E. Ozdaglar, D.P. Bertsekas, "Routing and wavelength assignment in optical networks," IEEE/ACM Transactions on Networking (TON), Vol. 11, no. 2, pp. 259- 272, April 2003.

[3] A. Wason, R. S. Kaler, "Wavelength Assignment Problem in Optical Networks," IJCSNS International Journal of Computer Science and Networks Security, Vol. 7, no. 4, pp. 27-31, April 2007.

[4] G.N. Rouskas, H.G. Perros. "A Tutorial on Optical Networks", Networking 2002 Tutorials, Lecture Notes in Computer Science, Vol. 2497, pp. 155-190, 2002.

[5] E. Lichtman, "Performance limitations imposed on all-optical ultralong lightwave systems at the zero-dispersion wavelength," Journal of Lightwave Technology, Vol. 13, no. 5, pp. 898-905, May 1995.

[6] S. Al Zahr, M. Gagnaire, N. Puech, and M. Koubaa, "Physical Layer Impairments in WDM Core Networks: a Comparison between a NorthAmerican Backbone and a Pan-European Backbone", Proceedings of the 2nd International Conference on Broadband Networks (Broadnets), Boston, Massachusetts, USA, Oct. 3-7, 2005.

[7] Y. Pointurier, M. Brandt-Pearce, T. Deng, and S. Subramaniam, "Fair QoS-aware adaptive routing and wavelength assignment in all-optical networks," IEEE International Conference on Communications, Vol. 6 , pp. 2433-2438, Istanbul-Turkey, June 2006.

[8] M. Menif, M. Karasek, K. Fouli, and L.A. Rusch, "Cross-gain modulation effect on the behaviour of packetized cascaded EDFAs," J. Opt. A: Pure Appl. Opt, Vol. 3, pp. 210-217, 2001.

[9] S. Pachnicke, T.Gravemann, M. Windmann and E. Voges, "Physically Constrained Routing in 10-Gb/s DWDM Networks Including Fiber Nonlinearities and Polarization Effects", IEEE Journal of Lightwave Technology, Vol. 24, pp. 3418-3426, 2006

[10] G.P. Agrawal, "Fiber-Optic Communications System," Third Edition 2002 John Wiley \& Sons, Inc

[11] Agilent Technologies, "Accurate Characterization of Source Spectra Using an Optical Spectrum Analyzer," Application Note 1550-5, May 2001.

[12] T. Antony, A. Gumaste, "WDM Network Design-1", Cisco Press, Cisco Feb 7, 2003.

[13] R. Essiambre, G. Raybon and B. Mikkelsen, "Optical Fiber Telecommunications", P. Kaminow, Academic Press, Vol. IVB, chap. 15 , pp. 725-861, 2002

[14] J. Redd, "Extinction Ratio and Power Penalty," Application Note HFAN-2.2.0. High-Frequency/Fiber Communications Group, Maxim Integrated Products.

[15] S. Pachnicke, J .Reichert, S. Spälter, and E. Voges. "Fast Analytical Assessment of the Signal Quality in Transparent Optical Networks", Journal of Lightwave Technology, Vol. 24, no. 2, February 2006

[16] D. Eppstein, "Finding the K-Shortest Paths," in IEEE Symposium on Foundations of Computer Science, pp. 154-165, 1994 